\title{
FLORESCIMENTO DA CANA-DE-AÇÚCAR: SEU CONTROLE COM ETHEPHON E SUA RELAÇÃO COM O ACÚMULO DE SACAROSE
}

\author{
R. DEUBER ${ }^{1}$ \\ M.V. CARLUCCI ${ }^{2}$ \\ 1 Engo Agro, CTC, Copersucar. \\ Engą Agrạ,, IAC. Instituto Agronômico, \\ Campinas.
}

\begin{abstract}
RESUMO
Instalou-se um experimento em cana-deaçúcar com as variedades SP70-1143, SP701284, IAC52/150, de $2^{\circ}$ corte, em que se aplicou ethephon, nas doses de 0,48 e 0,72 $\mathrm{kg} / \mathrm{ha}$, em cinco diferentes épocas, de 28 de janeiro a 15 de março de 1985. Foram obtidos os valores de pol, brix, fibra, açúcares redutores e pureza, mensalmente, de abril a agosto. Fez-se a contagem de flores emitidas neste último mês, e obteve-se a produção de colmos em outubro.

O ethephon inibiu totalmente a emissão da SP70-1143 e, parcialmente, da IAC52/150, até agosto. A SP70-1284 só floresceu $4 \%$. O florescimento de $50 \%$ dos colmos na SP70-1143 e 78\% na IAC52/150 não afetou o processo de acúmulo de sa carose. O ethephon também nao afetou esse processo em qualquer das variedades, mas reduziu o teor de fibras na SP70-1284, em julho, com quase todos os tratamentos. A produção de colmos nao foi afetada com os tratamentos realiza dos.
\end{abstract}

\section{SUMMARY}

A field experiment was carried out at the Copersucar Experimental
Station in Piracicaba with varieties SP701143, SP70-1284 and IAC-52/150, on second ratoon stage. Ethephon was applied at 0.48 and $0.72 \mathrm{~kg} / \mathrm{ha}$, at five different times from January, 28 to March 15, 1985. Values of pol, brix, fiber, purity and reducing sugars, were obtained monthly from April to August. Flower emission were counted in August and the number of stalks and yield were measure din October.

Ethephon caused complete inhibition of flowering in SP70 - 1143 and partial on IAC52/150 until August. SP70-1284 emited only $4 \%$ of flowers. Flowering of $50 \%$ for SP70-1143 and of 78\% for IAC52/150 did not affect the sucrose storage process. Ethephon did not affect this process either but reduced the fiber content in SP70-1284, in July, with almost all treatments. No differences for stalk numbers on yield were found among treatments.

\section{INTRODUÇÃO}

A cana-de-açúcar e uma gramínea de grande porte que pode florescer, ou não, em dado ano, dependendo das condições climáticas que 
ocorrem durante o período indutivo nesse ano.

O flor escimento da cana-de-açúcar tem sido encarado como prejudicial ao acúmulo de sacarose pois comumente aceito que a formação da flor requer grande quantidade de energia proveniente do colmo. Enquanto a maioria das gramíneas entra em processo de senescência a-pós a emissão das flores, a cana-de-açúcar, por ser planta de ciclo longo e possuir vasto sistema de reserva em seus colmos, apresenta um comportamento distinto.

Nas condições do Sudeste do Brasil e com as variedades aí cultivadas ocorrem, paralelamente, a formação deflores e o acúmulo de sa carose. A iniciação da infl orescência ocorre a partir de março com emissão de panícula de abril a maio. 0 acúmulo de sacarose acompanha esse processo, atingindo seu ponto mais elevado a partir de julho, de pendendo da variedade e época de plantio ou corte anterior. ALMEIDA et al. (1, 2), estudando esse processo com as variedades CP27-139, Co421 e Co331, verificaram que o ponto de máximo acúmulo de sacarose ocorreu quatro meses após aemis são das flores. Trabalhos mais re centes, conduzidos com as varie da des IAC48/65 (10), NA56-79 (5), SP 70-1078 e SP70-1143 (8), com níveis de florescimento variando de 50 a $86 \%$, tem confirmado que o pro cesso de matur ação ocorre, normalmente, por vários meses, apus o início da emissão de flores, sem aparentes prejuízos ao teor de sacarose.

Quando se procuram avaliar os efeitos do florescimento sobre a qualidade da matéria-prima, uma di ficuldade que surge é cons eguir ob ter, nas mesmas condições e ao mes mo tempo, colmos de cana-deaçúcar com e sem flores. Entre os regula- dores de crescimento que surgiram nos últimos anos destaca-se o ethe phon, por sua eficiência em inibir a forma ção de flores na cana-de-açúc ar $(3,5,11)$, tendo, portanto, potencial para prover a condição de colmos com e sem flores para estudos $(6,8)$. Por outro lado, o ethephon tem apresentado resultados variáveis em relação à riqueza de açúcar, elevando-a algumas vezes $(5,9)$, não a alterando em outras (8), redu zindo-a (4) ou, apres entando efeito de matura dor (12).

Com o objetivo de estudar os efeitos do ethephon sobre o flores cimento e qualidade da cana-de-açúcar, assim como os efeitos do florescimento sobre o acumulo de sacarose nos colmos, foi instalado um experimento de campo com aplicações de ethephon em diferentes épocas e doses.

\section{MATERIAL E MÉTODOS}

O experimento foi instalado na Estação Experimental Copersucar em Piracicaba-SP (CTEP), em uma area de Latossolo vermelho escuro.

As variedades estudadas foram SP701143, SP70-1284 e IAC52/150, plantadas em agosto de 1982, com cortes em agosto de 1983 e 1984. O espaçamento entre linhas era de $1,40 \mathrm{~m}$.

Os tratamentos consistiram na aplicação de ethephon $\mathrm{G}$ (ácido 2-cloroetilfosfônico) nas doses de 0,00; 0,48 e 0,72 kg de i.a. / ha, respectivamente, doses $\mathrm{D}_{0}, \mathrm{D}_{1}$ e $\mathrm{D}_{2}$. O produto aplicado é um concentra do emulsionvel, contendo $0,24 \mathrm{~kg}$ do i.a. por litro de formulação(1).

(1) Produto comercial Ethrel 2E. 
As aplicações foram feitas em 28 de janeiro de $1985\left(\mathrm{~T}_{1}\right), 12$ e 21 de fevereiro $\left(\mathrm{T}_{2}\right.$ e $\left.\mathrm{T}_{3}\right), 4$ e 15 de março $\left(\mathrm{T}_{4}\right.$ e $\left.\mathrm{T}_{5}\right)$. Utilizou-se um pulverizador costal de aço, impelido a $\mathrm{CO}_{2}$, munido de uma barra com quatro bicos 100.03, distanciados de $0,50 \mathrm{~m}$ dentro de cada par e de $1,40 \mathrm{~m}$ entre o centro dos pares de bicos, aplicando-se duas linhas de cada simultaneamente. o consumo de calda foi equival ente a 400 litros/ha.

O delineamento adotado foi ode parcelas subdivididas, sendo a dose a parcela e as épocas, as sub-parcelas, com duas repetições para cada tratamento. Cada subparcela, era formada por quatro linhas de dez metros de comprimento.

Foram feitas amostragens mensais, de abril até agosto, para análises tecnológicas, obtend o -se os valores de pol, brix, açúcares redutores, fibra e pureza. Em setembro fezse amostragem apenas da SP70-1143, analisando-se os mesmos parâmetros, separa damente, nos ter ços inferior, médio e superior. Cada amostra era formada por dez colmos seguidos em uma das linhas centrais da sub-parcela. De cada amostra foi obtido o peso antes das análises.

Foram feitas contagens de flores emitidas, em agosto, em uma linha inteira de 10 metros de cada sub-parcela.

Para avaliar a produção de col mos foi colhida e pesada uma linha de 10 metros de cada subparcela em 7 de outubro, sendo realizada a contagem do número de colmos dessa linha.

\section{RESULT ADOS E DISCUSS ÃO}

\section{Inibição do flores cimento}

O ano de 1985 foi favorável ao florescimento da cana-de-açúc ar na Região Sudeste do Brasil. A variedade SP70-1143 apresentou $50 \%$ de colmos com emissão de flores e a IAC52/150, 78\% em agosto e até ofi nal do ciclo (Quadro 1). A SP70 1284 é uma variedade não florífera nessa região e apenas $4 \%$ dos colmos flore sceram.

$\mathrm{O}$ ethephon foi eficiente em inibir totalmente a emissão de flo res na SP701143 , com todos os tra tamentos aplic ados. Para a IAC527 150, a eficiência não foi total, variando bastante entre os tratanentos. Verifica-se, entretanto, que na dose mais elevada, houve maior redução, sendo épocas T2 e T3, signific ativamente diferentes da teste munha. Nessa variedade o ethephon causou inibição inicial do florescimento, com a emissãodas Flores a partir de junho. Em agos to verificava-se grande número de Flores encartuchadas que assim per naneceram até o fim da colheita. Is so pode ser atribuído, em parte, á condição de seca ocorrida a partir de abril.

Os resultados obtidos indicam que a SP70-1143 é sensí vel à ação do ethephon, o que concorda com re sultados obtidos em outras condi ções (8).

\section{Qualidade da cana-de-açúcar}

Os valores de pol \% cana, amos tradas mensalmente, de abril à agosto, indicam que a aplicação do ethephon, em qualquer das épocas ou doses utilizadas, não afetou o acúmulo de sacarose. Os resulta dos obtidos com a dose $0,48 \mathrm{~kg} / \mathrm{ha}$ (D1) foram semelhantes aos obtidos com $0,72 \mathrm{~kg} / \mathrm{ha}$, não sendo represen tados por esse motivo. Nos tratamentos testemunha, das variedades SP70-1143 e IAC52/150, em que ocorreu o flore scimento, também não 
Quadro 1. Índice de florescimento (\%) no mês de agosto de 1985, no ensaio de ethephon em três variedades de cana na Estação Experi mental em Piracicaba. Médias de duas repetições.

\begin{tabular}{lcccc}
\hline & Data de & \multicolumn{3}{c}{ Florescimento } \\
\cline { 3 - 5 } aplicação & SP70-1143 & SP70-1284 & IAC52/150 \\
\hline $0,00 \mathrm{~kg} / \mathrm{ha}$ & - & 50 & 4 & $78 \mathrm{a}$ \\
$0,48 \mathrm{~kg} / \mathrm{ha}$ & $28 / 01$ & 0 & 0 & $41 \mathrm{ab}$ \\
& $12 / 02$ & 0 & 0 & $53 \mathrm{ab}$ \\
& $21 / 02$ & 0 & 0 & $35 \mathrm{ab}$ \\
& $04 / 03$ & 0 & 0 & $37 \mathrm{ab}$ \\
& $15 / 03$ & 2 & 1 & $48 \mathrm{ab}$ \\
& $28 / 01$ & 0 & 0 & $31 \mathrm{ab}$ \\
& $12 / 02$ & 0 & 0 & $16 \mathrm{~b}$ \\
& $21 / 02$ & 0 & 0 & $22 \mathrm{~b}$ \\
& $04 / 03$ & 0 & 0 & $30 \mathrm{ab}$ \\
& $15 / 03$ & 0 & 0 & $28 \mathrm{ab}$ \\
\hline D.M.S. (5\%) & & - & - & 54,69 \\
C.V. (\%) & & - & - & 25,10 \\
\hline
\end{tabular}

se verific ou alteração no acú mu- lo normal de sacarose, que ocorreu de modo igual aos tratamentos com ethephon. Para a SP70-1284, que não floresceu, não se observou qual quer diferença entre parcelas tratadas ou não com o ethephon, ocorrendo o mesmo padrao de acúmulo de sacarose das outras duas variedades.

A emissão de flores da IAC52/ 150, nas parcelas testemunhas, iniciou-se em abril e, da SP70-1143, em maio. Até o mês de setembro, para esta variedade e, ate o mês de agosto, para aquela, este fato não influiu no teor de sacarose, medido em pol \% cana (Quadro 4). Para a SP 70-1143 ess e acúmulo atingiu $17, \%$ de pol e $20,0 \%$ de brix, em setembro e, para a IAC52/150, esses valores chegaram a $16,5 \%$ e
$19,3 \%$, respectivamente, em agosto com ou sem formação de flores. Esses teores podem ser considerados elevados para as variedades em questão e foram atingidos em função de condições climáticas muito favoráveis a maturação da cana-de-açúcar ocorridas neste ano. Os resultados concordam com aqueles já encontrados por ALMEIDA et al. $(1,2)$ que também verificaram crescimento da pol \% cana em plantas florescidas das variedades CP27-139, Co421 e Co331 e, com outros autores $(5,8,10)$ que estudaram variedades atualmente plantadas.

Os valores de açúcar teórico recuperável (ATR), calculados apenas em agosto, foram proporcionais aos de pol \% cana, em todos os tratamentos indicando que nem o ethepon nem o florescimento afetaram 
significativamente as relações entre pol, fibra e pureza da cana.Para a SP7 0-1284, o valor de ATR chegou a $150 \mathrm{~kg} / \mathrm{t}$ de cana, o que pode ser considerado elevado para essa variedade. Os valores de ATR para as outras duas variedades são os nor malmente obtidos.

A paralisação do crescimento do colmo é um fator fundamental para favorecer o acúmulo de sacarose. Nes se sentido, tanto a formação da flor, a paralisação nat ural, quanto a aplica ção do ethephon, a artificial, resultaram no mesmo efeito favorável ã maturação da can a-de-açú car .

O teor de açúcares redutores (Quadro 2), no colmo inteiro, para a SP70-1284 apresentou reduções significativas em julho, em todos os tratamentos e, em agosto, na metade deles, principalmente com as épocas mais tardias. Para a SP701143 verificou-se apenas uma tendência de redução com as aplicações do ethephon. A analise das partes dos colmos (Quadro 4) da SP70-1143, em setembro, mostra que os menores teores de açúcares redutores estavam nos terços inferior e médio. Ess es dados não foram analisados estatisticamente por que não se obteve esse valor em to das as subparcelas testemunhas. No caso da IAC52/150 os teores desses açucares redutores foram mais elevados, em geral, nas parcel as tratadas, com algumas diferenças significa tivas em julho.

Valores menores de açúcares redutores indicam maior grau de matura ção e as aplicações mais tar dias modo geral, for am mais favoráveis para as duas variedades SP. A variedade 1AC52/150 parece que não foi afetada pelo regulador quanto à maturação. Os valores do A.R. apresentaram o mesmo padrão de decréscimo de maio a agosto nas três varie dades.

Das três varie dades estudadas, a SP70 1284 e a que possui menor teor de fibra sendo contudo a que apres entou resposta à aplica ção, com reduções significativas para diversos tratamentos (Quadro 4). Essas reduções, entretanto, não causaram elevações de pol \% ou de ATR, em qualquer dos tratamentos aplicados. Para a variedade SP70 1143, analisada em terços separados, no final do ciclo (Quadro 4), os valores de fibra não foram afetados, ao passo que para a IAC $52 / 150$, houve tendência de redução com a dose D2, a partir de junho.

A não alteração do teor de sacarose do colmo com a ocorrência do flore scimento $50 \%$ para a SP-70-1143 e $78 \%$ para a IAC52/150 - leva à conclusão que a exigência de energia requerida para a forma ção da haste floral é muito pequena, não resultando em redu ções perceptí veis da pol\% cana. Deve-se lembrar que a não formação de sementes, nas condições do Sudeste do Brasil, é que resulta nessa não alteração do teor de sacarose. A haste floral, sem sementes, representa muito pouco em termos de matéria seca produzida.

As produções de colmos, apre sentavam valores que podem ser considerados bons para o terc eiro corte - $96 \mathrm{t} / \mathrm{ha}$ para a SP70 $1143,75 \mathrm{t} / \mathrm{ha}$ para a SP70-1284 e $83 \mathrm{t} /$ ha para a IAC52/150 - não ocorrendo diferenças significativas entre os tratamentos, nem para produções nem para o número de colmos/área (Quadro 5). 
Quadro 2. Valores mensais de açücares redutores, obtidos no experimento de campo, em cana-de-açūcar, $\underset{N}{\omega}$ com aplicações de ethephon, na Estação Experimental Copersucar em Piracicaba. Média duas repetições.

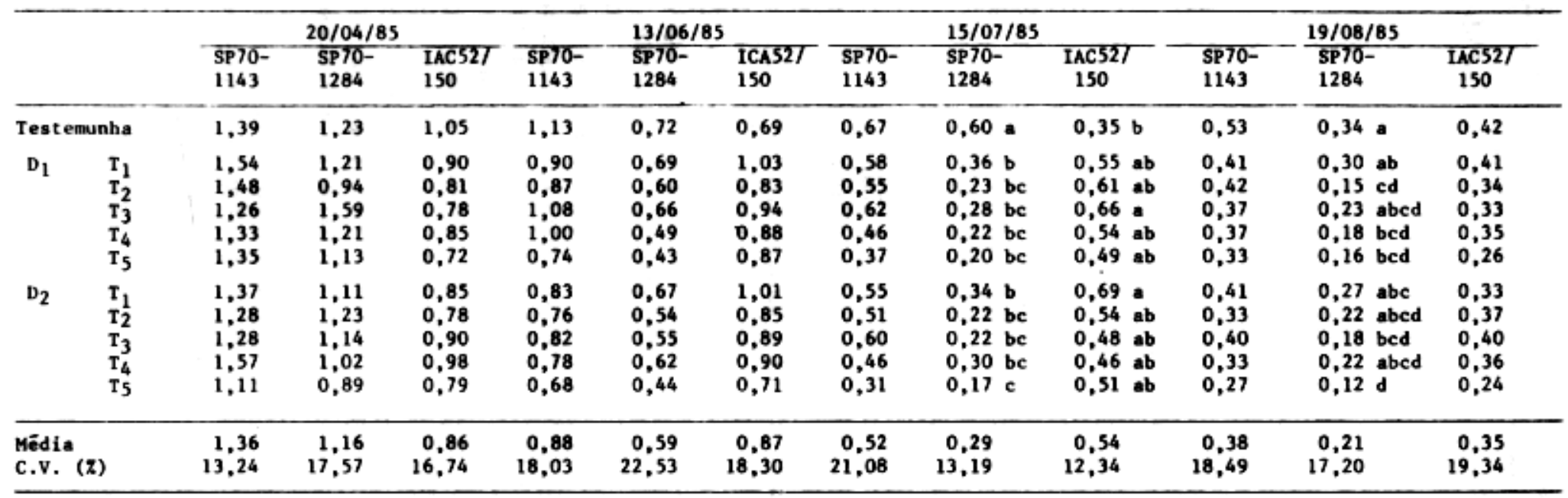

Obs.: Valores nas colunas seguidos de letras iguais, näo diferem entre si, ao nivel de $5 z$ (teste Tukey). Nas demais colunas näo ocorre ram diferenças significativas.
$D_{1}=0,48 \mathrm{~kg} / \mathrm{ha}$ de ethephon
$\mathrm{T}_{1}=$ apl icado em 28/01
$T_{3}$ - aplicado em 21/02
$T_{4}=$ aplicaçäo em 04/03
$\mathrm{T}_{5}$ - aplicaçao em 15/03 
Quadro 3. Valores mensais de fibra, obtidos no experimento de campo, em cana-de-açūcar, com aplica çōes de ethephon, na Estação Experimental Copersucar em Piracicaba. Médias de duas repetî̉ çōes.

\begin{tabular}{|c|c|c|c|c|c|c|c|c|c|c|c|c|c|}
\hline & & \multicolumn{3}{|c|}{$20 / 05 / 85$} & \multicolumn{4}{|c|}{$13 / 06 / 85$} & \multicolumn{2}{|l|}{$15 / 07 / 85$} & \multicolumn{3}{|c|}{$19 / 08 / 85$} \\
\hline & & $\begin{array}{r}\text { SP70- } \\
1143\end{array}$ & $\begin{array}{r}\text { SP70- } \\
1284\end{array}$ & $\begin{array}{c}\text { IACS2/ } \\
150\end{array}$ & $\begin{array}{r}\text { SP70- } \\
1143\end{array}$ & $\begin{array}{r}\text { SP70- } \\
1284\end{array}$ & $\begin{array}{l}\text { IAC52/ } \\
150\end{array}$ & $\begin{array}{r}\text { SP70- } \\
1143\end{array}$ & $\begin{array}{r}\text { SP70- } \\
1284\end{array}$ & $\begin{array}{l}\text { IACS2/ } \\
150\end{array}$ & $\begin{array}{r}\text { SP70- } \\
1143\end{array}$ & $\begin{array}{r}\text { SP70- } \\
1284\end{array}$ & $\begin{array}{l}\text { LAC52/ } \\
150\end{array}$ \\
\hline \multicolumn{2}{|c|}{ Testemunha } & 11,78 & $10,63 a$ & 11,78 & 12,39 & $10,83 a$ & 13,00 & 13,58 & $11,05 \mathrm{a}$ & 13,83 & 14,08 & 11,60 & 13,88 \\
\hline $\mathrm{D}_{1}$ & $\begin{array}{l}\mathrm{T} 1 \\
\mathrm{~T}_{2} \\
\mathrm{~T}_{3} \\
\mathrm{~T}_{4} \\
\mathrm{~T} 5\end{array}$ & $\begin{array}{l}10,92 \\
12,16 \\
11,71 \\
10,76 \\
12,01\end{array}$ & $\begin{array}{l}9,29 \mathrm{~b} \\
9,46 \mathrm{ab} \\
9,07 \mathrm{~b} \\
9,67 \mathrm{ab} \\
9,03 \mathrm{~b}\end{array}$ & $\begin{array}{l}11,91 \\
11,74 \\
11,95 \\
12,11 \\
11,62\end{array}$ & $\begin{array}{l}10,91 \\
11,93 \\
11,66 \\
12,25 \\
12,16\end{array}$ & $\begin{array}{l}9,21 \mathrm{~b} \\
9,67 \mathrm{ab} \\
9,18 \mathrm{~b} \\
9,86 \mathrm{ab} \\
9,02 \mathrm{~b}\end{array}$ & $\begin{array}{l}12,33 \\
12,52 \\
12,67 \\
11,56 \\
11,67\end{array}$ & $\begin{array}{l}11,97 \\
12,30 \\
12,80 \\
13,34 \\
12,63\end{array}$ & $\begin{array}{r}9,28 \mathrm{~b} \\
10,15 \mathrm{ab} \\
9,57 \mathrm{~b} \\
10,45 \mathrm{ab} \\
9,58 \mathrm{~b}\end{array}$ & $\begin{array}{l}12,10 \\
12,12 \\
12,03 \\
12,43 \\
12,00\end{array}$ & $\begin{array}{l}12,65 \\
13,87 \\
14,07 \\
13,96 \\
13,94\end{array}$ & $\begin{array}{l}10,65 \\
10,71 \\
10,63 \\
11,39 \\
10,21\end{array}$ & $\begin{array}{l}13,49 \\
13,73 \\
13,07 \\
14,92 \\
12,92\end{array}$ \\
\hline D2 & $\begin{array}{l}\mathbf{T}_{1} \\
\mathbf{T}_{2} \\
\mathbf{T}_{3} \\
\mathbf{T}_{4} \\
\mathrm{~T}_{5}\end{array}$ & $\begin{array}{l}11,12 \\
12,05 \\
11,58 \\
11,73 \\
12,55\end{array}$ & $\begin{array}{l}9,27 \mathrm{~b} \\
9,27 \mathrm{~b} \\
9,69 \mathrm{ab} \\
9,52 \mathrm{ab} \\
9,43 \mathrm{~b}\end{array}$ & $\begin{array}{l}11,32 \\
11,66 \\
11,75 \\
12,02 \\
11,23\end{array}$ & $\begin{array}{l}11,87 \\
11,46 \\
11,67 \\
11,70 \\
12,04\end{array}$ & $\begin{array}{l}9,80 \mathrm{ab} \\
9,46 \mathrm{ab} \\
9,66 \mathrm{ab} \\
9,65 \mathrm{ab} \\
9,44 \mathrm{ab}\end{array}$ & $\begin{array}{l}12,75 \\
12,28 \\
11,77 \\
12,17 \\
11,50\end{array}$ & $\begin{array}{l}12,62 \\
11,58 \\
13,09 \\
12,65 \\
12,60\end{array}$ & $\begin{array}{l}9,63 \mathrm{~b} \\
9,42 \mathrm{~b} \\
9,66 \mathrm{~b} \\
9,67 \mathrm{~b} \\
9,48 \mathrm{~b}\end{array}$ & $\begin{array}{l}12,35 \\
12,47 \\
11,85 \\
11,84 \\
11,81\end{array}$ & $\begin{array}{l}12,97 \\
12,61 \\
13,56 \\
13,82 \\
13,71\end{array}$ & $\begin{array}{l}10,60 \\
10,38 \\
10,05 \\
10,54 \\
10,39\end{array}$ & $\begin{array}{l}13,11 \\
12,11 \\
12,14 \\
12,86 \\
12,19\end{array}$ \\
\hline \multicolumn{2}{|c|}{ Média } & 11,63 & 9,47 & 11,74 & 11,84 & 9,62 & 12,21 & 12,65 & 9,86 & 12,26 & 13,37 & 10,65 & 13,13 \\
\hline \multicolumn{2}{|c|}{ C.V. (z) } & 4,27 & 3,06 & 5,16 & 3,84 & 4,20 & 3,96 & 4,10 & 3,06 & 4,27 & 6,36 & 4,72 & 5,37 \\
\hline
\end{tabular}

Obs.: Valores nas colunas seguidos de letras iguais, näo diferem entre si, ao nível de Sz (Teste Tukey). Nas demais colunas näo ocorreram diferenças significativas. 
Quadro 4. Valores de fibra, açúcares redutores, pol e ATR, obtidos no experimento de campo, em cana-de-açúcar, com aplicações de ethephon, na Estação Experimental Copersucar em Piracicaba, variedade SP70-1143, 16/09/87. Mëdias de duas repetições.

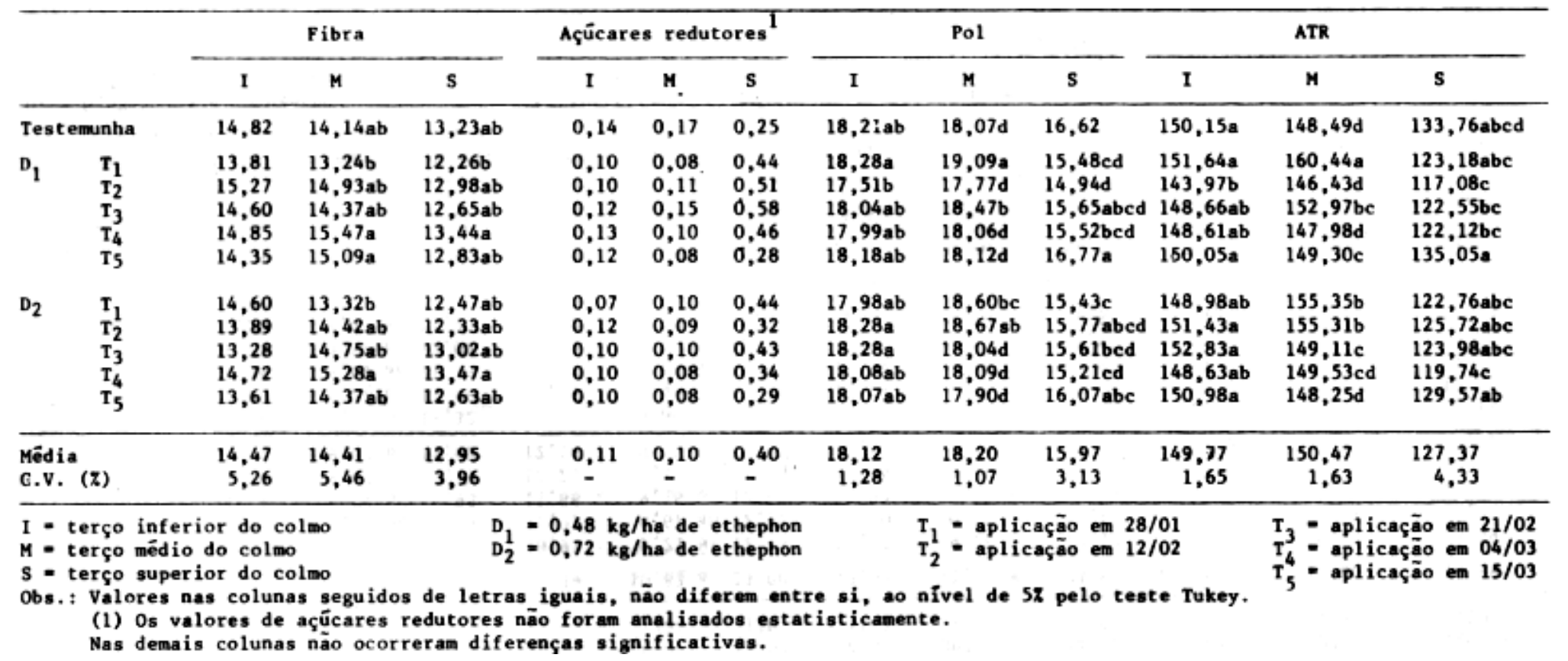


Quadro 5. Número e peso de colmos em kg por parcela no ensaio de aplicação de ethephpn em três variedades de cana-de-açúcar, na Estação Experimental Copersucar em Piracicaba, em 07/10 / 1985. Médias de duas repetições de $14 \mathrm{~m}^{2}$.

\begin{tabular}{|c|c|c|c|c|c|c|c|c|c|c|}
\hline \multirow{2}{*}{ Dose } & \multirow{2}{*}{$\begin{array}{c}\text { Data } \\
\text { de } \\
\text { aplic. }\end{array}$} & \multicolumn{3}{|c|}{ SP70-1143 } & \multicolumn{3}{|c|}{ SP70-1284 } & \multicolumn{3}{|c|}{ IAC52/150 } \\
\hline & & Nümero & Peso & kg/colmo & Número & Peso & $\mathrm{kg} / \mathrm{colmo}$ & Número & Peso & $\mathrm{kg} / \mathrm{colmo}$ \\
\hline $0,0 \mathrm{~kg} / \mathrm{ha}$ & - & 129 & 135 & 1,05 & 109 & 105 & 0,96 & 126 & 116 & 0,92 \\
\hline $0,48 \mathrm{~kg} / \mathrm{ha}$ & $\begin{array}{l}28 / 01 \\
12 / 02 \\
21 / 02 \\
04 / 03 \\
15 / 03\end{array}$ & $\begin{array}{l}128 \\
117 \\
114 \\
109 \\
111\end{array}$ & $\begin{array}{l}167 \\
107 \\
129 \\
127 \\
120\end{array}$ & $\begin{array}{l}1,30 \\
0,92 \\
1,12 \\
1,17 \\
1,08\end{array}$ & $\begin{array}{r}97 \\
116 \\
104 \\
101 \\
110\end{array}$ & $\begin{array}{l}109 \\
109 \\
108 \\
103 \\
108\end{array}$ & $\begin{array}{l}1,12 \\
0,93 \\
1,04 \\
1,02 \\
0,98\end{array}$ & $\begin{array}{l}117 \\
125 \\
144 \\
130 \\
122\end{array}$ & $\begin{array}{l}109 \\
134 \\
135 \\
112 \\
103\end{array}$ & $\begin{array}{l}0,93 \\
1,07 \\
0,94 \\
0,86 \\
0,84\end{array}$ \\
\hline $0,72 \mathrm{~kg} / \mathrm{ha}$ & $\begin{array}{l}28 / 01 \\
12 / 02 \\
21 / 02 \\
04 / 03 \\
15 / 03\end{array}$ & $\begin{array}{l}102 \\
116 \\
139 \\
129 \\
126\end{array}$ & $\begin{array}{l}130 \\
141 \\
140 \\
130 \\
128\end{array}$ & $\begin{array}{l}1,27 \\
1,21 \\
1,00 \\
1,01 \\
1,02\end{array}$ & $\begin{array}{l}112 \\
114 \\
113 \\
128 \\
126\end{array}$ & $\begin{array}{l}117 \\
125 \\
143 \\
132 \\
138\end{array}$ & $\begin{array}{l}1,04 \\
1,10 \\
1,26 \\
1,03 \\
1,09\end{array}$ & $\begin{array}{l}114 \\
103 \\
131 \\
125 \\
123\end{array}$ & $\begin{array}{r}94 \\
101 \\
135 \\
139 \\
118\end{array}$ & $\begin{array}{l}0,82 \\
0,98 \\
1,02 \\
1,11 \\
0,96\end{array}$ \\
\hline $\begin{array}{l}\text { Média } \\
\text { C.v. (z) }\end{array}$ & & $\begin{array}{r}120,0 \\
8,12\end{array}$ & $\begin{array}{r}119,4 \\
16,8\end{array}$ & $\begin{array}{r}1,10 \\
411,00\end{array}$ & $\begin{array}{r}111,8 \\
8,46\end{array}$ & $\begin{array}{r}117,9 \\
12,0\end{array}$ & $\begin{array}{ll}9 & 1,05 \\
8 & 8,62\end{array}$ & $\begin{array}{r}123,6 \\
8,43\end{array}$ & $\begin{array}{r}117,8 \\
13,38\end{array}$ & $\begin{array}{l}0,95 \\
9,67\end{array}$ \\
\hline
\end{tabular}

Obs.: Não foram obtidas diferenças significativas para esses valores. 


\section{LITBRATURA CITADA}

1. Almeida, J.R., Valsechi, 0 . \& Pimentel Gomes, F. 0 florescimento da cana-de - açúcar. E.S.A. "Luiz de Queiroz", Anais, 2:49-117, 1945.

2. Almeida, J.R.; Val sechi, 0.; Leme Jr., J.; Pimentel Gomes, F.; Cardoso, E. de M. \& Camolese, N. O florescimento na variedade de cana Co331. E.S.A. "Luiz de Quei roz", Anais, 9: $157-174$, 1952 .

3. Carr, J.; Moore, P.H. \& Osgood, R.V. Tassel prevention by ethephon and diquat. Unpublished HSPA data.

4. Clowes, M.S.T.J. Growth stimu lation from ethrel and the effects of gibberelin and when applied to sugarcane. The South African Sugar Technologists Assoc. Proceedings, 1980. p.146-150.

5. Coletti, J.T.; Lorenzeti, J. T.; Lorenzetti, J.M.; Freitas, P.G.R.; Corbini, J.L.; Walter, L.A.M. \& Camponez Ne to, A. A inibição de $f 10^{-}$ rescimento pelo uso do ethe phon e sua influência na biō massa. Cong. Nacional da STAB, 3. Convenção da ACTALAC, 5., 1984. Anais. p.348 -351 .

6. Deuber, R. Florescimento e ma turação da cana-de-açūcar . Seminário de Tecnologia Copersucar , 3, 1986. p. 585593.
7. Deuber, R. \& Chalita, R. Rela törio de testes com reguladores de crescimento em cana-de-açücar no ano de 1984. Copersucar, 1985. 14pp. (mi meo).

8. Deuber, R. \& Irvine, J.E. Controle do florescimento da cana-de-açúcar com aplicação de ethephon. Boletim Técnico Copersucar, 36: 16$24,1986$.

9. Ide, B.Y. \& Chalita, E. Efeito do ethephon no desenvolvimento da cana-de-açūcar florescimento e maturação. Boletim Técnico Copersucar, 29: 26-34, 1985.

10. Nunes Jr., D.; Giacomini, G.S. \& Oliveira, A.A. Comparação do florescimento, isoporiza ção e qualidade tecnolögica em duas variedades de ca na-de-açúcar na presença de maturador. Bolet im Técnico Copersucar, 20: 20-31, 1982.

11. Osgood, R.; Moore, P.R. \& Carr, $\mathrm{J}$. Comparation of diquat and ethephon for prevention of flower initiation in sugarcane (Saccharum spp. hybrids). Plant Growth Regulator Society Meet, 1983.

12. Yang, P.C. \& Ho, F.W. Effects of embark and ethrel on sugarcane quality, yields an ratoon regrowth. ISSCT, 1980, 1: 711-723, Procee dings. 